\title{
Impact of Glycemic Control on the Clinical Outcome in Diabetic Patients With Percutaneous Coronary Intervention
}

\author{
- From the FU-Registry -
}

Amane Ike, MD; Hiroaki Nishikawa, MD, PhD; Kazuyuki Shirai, MD, PhD; Ken Mori, MD; Takashi Kuwano, MD; Yusuke Fukuda, MD, PhD; Yosuke Takamiya, MD, PhD; Daizaburo Yanagi, MD, PhD; Kazumitsu Kubota, MD; Yoshihiro Tsuchiya, MD, PhD; Bo Zhang, PhD; Shin-ichiro Miura, MD, PhD; Keijiro Saku, MD, PhD

\begin{abstract}
Background: It is not yet clear whether glycemic control affects the clinical outcome of percutaneous coronary intervention $(\mathrm{PCl})$ in diabetic patients.

Methods and Results: This study compared the effects of glycemic control on the clinical outcome in 2 groups of patients with diabetes mellitus (DM) who underwent PCl: a poor-glycemic-control group, who showed greater than $6.9 \% \mathrm{HbA}_{1 \mathrm{c}}$ at the time of $\mathrm{PCl}$ (Pre-HbA $\mathrm{H}_{1 \mathrm{c}}$ ) (' $\geq 6.9$ group', $\mathrm{n}=334$ patients) and a good-glycemic-control group, who showed less than $<6.9 \%$ at Pre-HbA 1 ( ' $<6.9$ group', $n=212$ patients). The patients in the $\geq 6.9$ group were further divided into 2 groups for further comparisons: a 'DM control group' and a 'Poor control group'. At follow-up (300 days), the incidence of major adverse cardiac event (MACE) was significantly $(P<0.05)$ lower in the $<6.9$ group (18.4\% vs. $26.2 \%$ ). However, there was no difference in MACE between the DM control group and the Poor control group. In a multivariate analysis, there was no relationship between the incidence of MACE and Pre$\mathrm{HbA}_{1 \mathrm{c}}$, Pre-HbA $\mathrm{H}_{1 \mathrm{c}} \geq 6.9 \%$ or the $\mathrm{HbA}_{1 c}$ difference (Pre-HbA $\mathrm{H}_{1 \mathrm{c}}-\mathrm{HbA}_{1 \mathrm{c}}$ at follow-up).
\end{abstract}

Conclusions: Clinical outcomes in the $<6.9$ group were superior to those in the $\geq 6.9$ group as pre- $\mathrm{PCl}$ glycemic control affected the baseline characteristics. The results suggested that glycemic control started at $\mathrm{PCl}$ was not associated with an improvement in the clinical outcome at follow-up. (Circ J 2011; 75: 791-799)

Key Words: Clinical outcome; Diabetes mellitus; Glycemic control; Percutaneous coronary intervention

I t has been established that glycemic control can, in the long term, prevent the occurrence of cardiovascular diseases (CVD) in patients with diabetes mellitus (DM), as demonstrated in the United Kingdom Prospective Diabetes Study (UKPDS) 80, ${ }^{1}$ the Framingham Heart Study, ${ }^{2}$ and other studies. In addition, DM patients undergoing percutaneous coronary intervention (PCI) are more likely to develop stent restenosis as well as major adverse cardiovascular events (MACE), and have a worse clinical outcome in both the short and long term than non-DM patients undergoing PCI. ${ }^{3-5}$ These trends have also been observed in cases of acute myocardial infarction (MI), and DM is associated with rehospitalization for heart failure ${ }^{6}$ after PCI. These differences have been reported to be due to chronic hyperglycemia, because it induces vascular endothelial injury, ${ }^{7}$ inflammatory reactions, ${ }^{8}$ reactive oxygen species or advanced glycation end products, which leads to accelerated cell pro- liferation ${ }^{9}$ or other pathological conditions such as in the blood-clotting system or fibrinolysis. ${ }^{10}$ Thus, we have to overcome such problems in DM patients undergoing PCI, although the rate of stent restenosis has decreased since the introduction of drug-eluting stents (DES). ${ }^{11}$ The incidence of stent restenosis and/or MACE can be reduced by intensive glycemic control in diabetic patients, ${ }^{12}$ while the Diabetes Control and Complications Trial (DCCT) and Epidemiology of Diabetes Interventions and Complications (EDIC) and UKPDS80 studies have reported that due to "metabolic memory (Legacy)' effects, intensive glycemic control can not readily reduce the occurrence of CVD. ${ }^{1,13,14}$ Is it possible that glycemic control started at the time of PCI and continued afterwards might prevent stent restenosis and MACE in Japanese diabetic patients?

Received May 18, 2010; revised manuscript received September 25, 2010; accepted September 30, 2010; released online March 18,

2011 Time for primary review: 27 days

Department of Cardiology, Fukuoka University School of Medicine, Fukuoka, Japan

Mailing address: Keijiro Saku, MD, Department of Cardiology, Fukuoka University School of Medicine, 7-45-1 Nanakuma, Jonan-ku,

Fukuoka 814-0180, Japan. E-mail: saku-k@cis.fukuoka-u.ac.jp

ISSN-1346-9843 doi:10.1253/circj.CJ-10-0474

All rights are reserved to the Japanese Circulation Society. For permissions, please e-mail: cj@j-circ.or.jp 


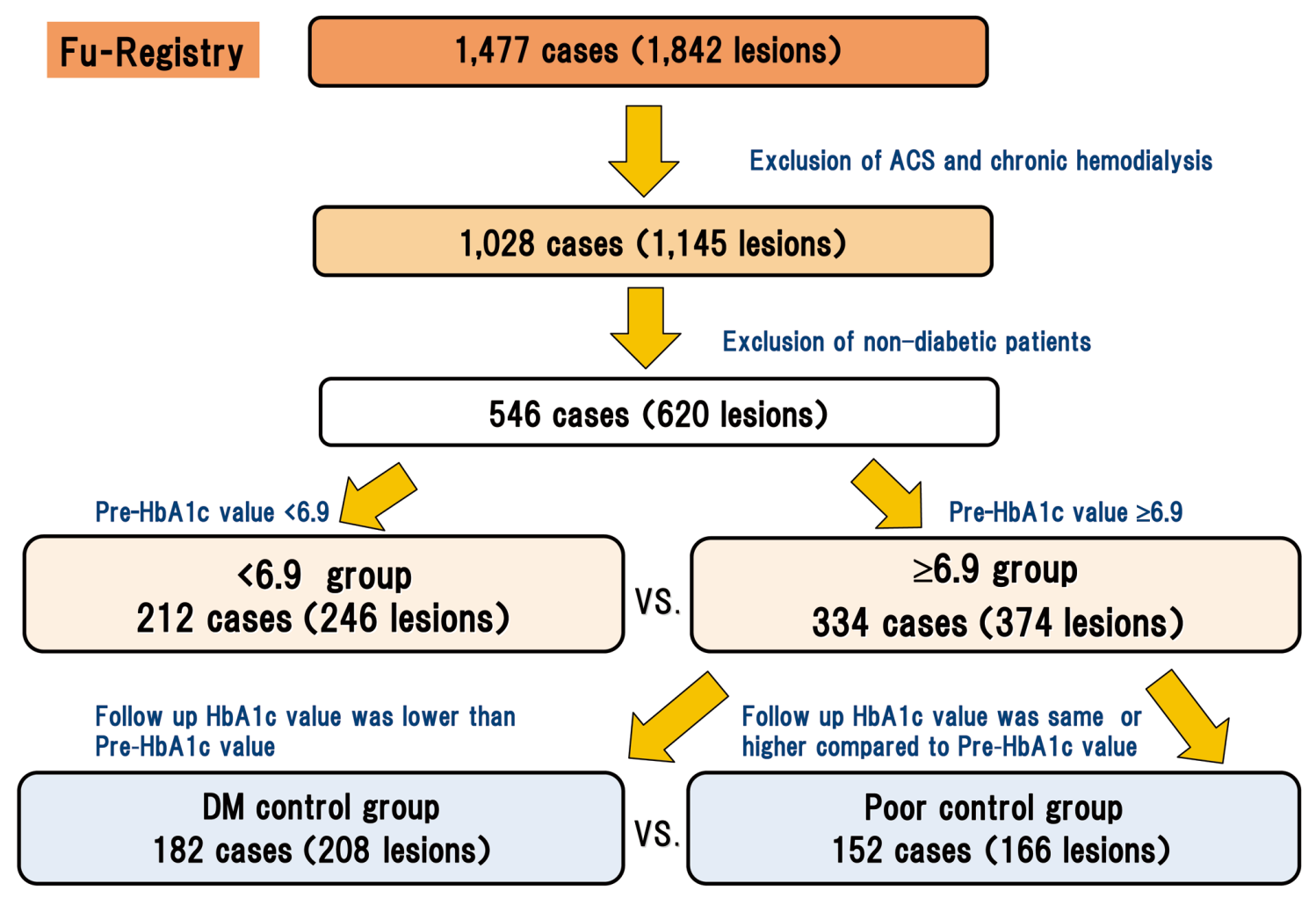

Figure. Outline of the study (the FU-Registry). HbA1c values: we used the National Glycohemoglobin Standardization Program (NGSP) value.

\section{Editorial p 773}

In this study, we conducted a comparative investigation including an angiographical analysis, from the Fukuoka University Registry (FU-Registry), on the effects of glycemic control on the clinical outcome at approximately 300 days of follow-up as assessed by $\mathrm{HbA} 1 \mathrm{c}$, when such control was started before PCI and continued afterwards.

\section{Methods}

\section{Patient Selection}

This study was conducted at Fukuoka University Hospital, Fukuoka University Chikushi Hospital and Fukuoka Hakujuji Hospital, and the analysis was performed using our database of FU-Registry. This study was conducted in accordance with the Declaration of Helsinki, and approval was obtained from the Independent Review Board of Fukuoka University Hospital. Among 1,477 patients (1,842 lesions) who underwent PCI and received stent placement between January 2003 and July 2008, we considered 1,028 patients (1,145 lesions) after excluding those with acute coronary syndrome and those with chronic hemodialysis (Figure). We further selected 546 DM patients from among these 1,028 patients and divided them into 2 groups according to the $\mathrm{HbA}_{1 c}$ value at the time of PCI (Pre-HbAic). The HbAic value used in this study was recalculated using the National Glycohemoglobin Standardization Program (NGSP) $)^{15}$ by adding $0.4 \%$ to previous values used in Japan, and a cutoff value of Pre-HbA 1 c $6.9 \%$ (previously $6.5 \%$ ) was the target level for patients with ischemic heart disease as defined by the Japanese Circulation Society. ${ }^{16}$ The 2 groups were a poor-BS-control group, who had Pre-HbAlc values greater than $6.9 \%$ (' $\geq 6.9$ group', $n=334$ patients [374 lesions]) and a relatively a good-BS-control group, who had Pre-HbAic values below $6.9 \%$ ( $<6.9$ group', $\mathrm{n}=212$ patients [246 lesions]). We conducted a comparative investigation of the patients' follow-up clinical outcomes that included an analysis of angiographical data. The patients in the $\geq 6.9$ group were further divided into 2 groups: a 'DM control group', who received better glycemic control before and after PCI, and who presented lower Pre-HbAlc values at the follow-up ( $\mathrm{n}=182$ patients [208 lesions]) and a 'Poor control group', who showed the same or higher values compared to Pre-HbAlc $(\mathrm{n}=152$ patients [166 lesions]) (Figure).

Ninety-five percent of the total subjects participated in the clinical follow-up at approximately 300 days. The presence of MACE was investigated at the last visit to the hospital and by telephone queries, while angiographic follow-up was conducted in $80 \%$ of the total participants.

For the definition of DM, we adopted the classification and diagnostic criteria of the Japan Diabetes Society; ie, either a fasting plasma glucose cutoff concentration of $\geq 126 \mathrm{mg} / \mathrm{dl}$, a plasma glucose concentration of $\geq 200 \mathrm{mg} / \mathrm{dl}$ at $2 \mathrm{~h}$ after a $75 \mathrm{~g}$ oral glucose tolerance test, or a casual plasma glucose $\geq 200 \mathrm{mg} / \mathrm{dl}$ (the cutoff values represent venous plasma glucose concentrations) was regarded as indicative of DM. We also included patients who were receiving continued oral administration of hypoglycemic agents (eg, insulin, sulfonylurea, etc), or who had evidently been diagnosed 


\begin{tabular}{|c|c|c|c|c|}
\hline & \multirow[b]{2}{*}{$<6.9$ group } & \multicolumn{3}{|c|}{$\geq 6.9$ group } \\
\hline & & Total & $\begin{array}{l}\text { DM control } \\
\text { group }\end{array}$ & $\begin{array}{l}\text { Poor control } \\
\text { group }\end{array}$ \\
\hline $\mathbf{N}$ & 212 & 334 & 182 & 152 \\
\hline Mean age (years) & $68.0 \pm 10.0$ & $66.7 \pm 9.3$ & $65.8 \pm 9.1$ & $67.9 \pm 9.4^{t \dagger}$ \\
\hline Male (\%) & 73.6 & 76.3 & 75.9 & 77.0 \\
\hline BMI $\left(\mathrm{kg} / \mathrm{m}^{2}\right)$ & $24.2 \pm 3.8$ & $24.3 \pm 3.7$ & $24.3 \pm 3.6$ & $24.3 \pm 3.8$ \\
\hline UCG-LVEF (\%) & 60.4 & 60.3 & 61.0 & 59.5 \\
\hline \multicolumn{5}{|c|}{ Prior diseases/complicated diseases } \\
\hline Prior MI (\%) & 34.4 & 29.9 & 28.0 & 32.2 \\
\hline Prior CABG (\%) & 1.9 & $7.8^{\star \star}$ & 7.1 & 8.6 \\
\hline 3-VD (\%) & 15.6 & $23.7^{\star}$ & 23.6 & 23.7 \\
\hline Hypertension (\%) & 78.3 & $69.8^{\star}$ & 75.8 & $62.5^{\ddagger}$ \\
\hline Hyperlipidemia (\%) & 67.9 & 73.7 & 81.3 & 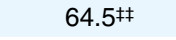 \\
\hline \multicolumn{5}{|l|}{ Blood sampling test } \\
\hline $\mathrm{Cr}(\mathrm{mg} / \mathrm{dl})$ & $1.1 \pm 0.6$ & $1.0 \pm 0.5^{\star \star}$ & $1.0 \pm 0.5$ & $0.9 \pm 0.4$ \\
\hline $\mathrm{TC}(\mathrm{mg} / \mathrm{dl})$ & $179.3 \pm 42.0$ & $189.1 \pm 37.6^{*}$ & $192.0 \pm 40.1$ & $185.6 \pm 33.9$ \\
\hline $\mathrm{TG}(\mathrm{mg} / \mathrm{dl})$ & $135.7 \pm 109.2$ & $146.9 \pm 98.4$ & $148.4 \pm 98.3$ & $145.2 \pm 98.7$ \\
\hline HDL-C (mg/dl) & $45.6 \pm 12.4$ & $45.7 \pm 12.4$ & $45.5 \pm 12.4$ & $45.9 \pm 12.6$ \\
\hline Pre-HbA $1 c(\%)$ & $6.3 \pm 0.4$ & $8.2 \pm 1.2^{\dagger}$ & $8.4 \pm 1.3$ & $7.9 \pm 1.0 \ddagger \ddagger$ \\
\hline
\end{tabular}

$<6.9$ group vs. $\geq 6.9$ group: $P$-value ${ }^{*}<0.05,{ }^{* *}<0.01,{ }^{\dagger}<0.001$.

DM control group vs. Poor control group: $P$-value $t+<0.05, \ddagger<0.01, \neq \neq<0.001$.

DM, diabetes mellitus; BMI, body mass index; MI, myocardial infarction; CABG, coronary artery bypass graft; 3-VD,

3 vessel disease; TC, total cholesterol; HDL-C, high density lipoprotein-cholesterol.

$\mathrm{HbA}{ }_{1 c}$ values: we used the National Glycohemoglobin Standardization Program (NGSP) ${ }^{15}$ value.

as DM. MACE was defined to include death from all causes, MI, and target lesion revascularization (TLR).

The definition of MI included both ST-T elevation and non-ST-T elevation.

For a diagnosis of MI, the patient had to have met either of the following criteria: evident ischemic electrocardiogram changes or elevation of cardiac biomarker concentrations (rapid troponin $\mathrm{T}$ test positive, creatine kinase (CK) more than twice the normal value, CK-MB exceeding the upper limit of the standard value.

Almost all of the blood samples were drawn after an overnight fast, because the samples were essentially based on inpatient data.

\section{$\mathrm{PCI} /$ Intravascular Ultrasound (IVUS) Procedure}

We performed PCI on patients who were angiographically observed to have a lesion with more than $50 \%$ stenosis, on the condition that the patient had a chest symptom or that the presence of ischemia was evident from the results of a noninvasive examination (treadmill electrocardiogram, myocardial perfusion scintigraphy). Procedural endpoints included no dissections to induce blood-flow obstruction, achievement of Thrombolysis In MI (TIMI) 3 flow, and angiographic stenosis rate $\geq 10 \%$. IVUS was performed at the operator's discretion in approximately one-third of the total participants. We did not include pre-procedural IVUS data in the analysis because in some cases the IVUS catheter did not pass through the lesions. Thus, only post-procedural IVUS data were used in the present analysis.

\section{Medication (Antiplatelets)}

The administration of antiplatelets (100 mg Aspirin plus $200 \mathrm{mg}$ Ticlopidine or $75 \mathrm{mg}$ Clopidogrel) was started in all patients at least $48 \mathrm{~h}$ prior to stent placement. Basically, the administration of antiplatelets except Aspirin was to be continued for at least 2 weeks after PCI for cases with a bare-metal stent (BMS). For cases with DES, the administration of all antiplatelets was to be continued for at least 9 months after PCI.

\section{Quantitative Coronary Angiography (QCA)}

QCA was randomly performed in approximately $45 \%$ of the total patients. Quantitative and qualitative analyses were conducted in our core laboratory at Fukuoka University, Fukuoka, Japan, using a CMS-GFT system (Medis, Leiden, the Netherlands) as described previously. ${ }^{17-19}$ Pre- and post-procedural angiograms as well as follow-up angiograms were analyzed. All measurements were performed with images after the intracoronary administration of nitroglycerin. The in-lesion segment was defined to be measured from $5 \mathrm{~mm}$ outside of the proximal and distal edges of the stent or in-stent. Late loss was defined as the difference in minimum lesion diameter (MLD) between post-procedural and follow-up angiograms. We defined restenosis as the recurrence of stenosis at a rate of more than $50 \%$, as described previously. ${ }^{17,18}$

\section{Statistical Analysis}

Statistical analysis was performed using SAS software, version 9.1 (SAS Institute, Cary, NC, USA) at Fukuoka University. The chi-square test was used for intergroup comparisons of categorical variables. For intergroup comparisons of continuous variables, we used Wilcoxon's rank-sum test and Student's t-test. The values are expressed as mean \pm STD. Statistical significance was defined as a P-value less than 0.05 . We used a multiple logistic regression analysis for the multivariate analysis.

\section{Results}

Table 1 shows the patient characteristics for the good-glycemic-control group [Pre-HbA1c values below 6.9\% $(<6.9$ 
Table 2. Medications at $\mathrm{PCl}$ and Follow-up

\begin{tabular}{|c|c|c|c|c|c|c|c|c|}
\hline & \multicolumn{4}{|c|}{ Medications at $\mathrm{PCl}$} & \multicolumn{4}{|c|}{ Medications at follow-up } \\
\hline & \multirow[b]{2}{*}{$\begin{array}{c}<6.9 \\
\text { group }\end{array}$} & \multicolumn{3}{|c|}{$\geq 6.9$ group } & \multirow[b]{2}{*}{$\begin{array}{c}<6.9 \\
\text { group }\end{array}$} & \multicolumn{3}{|c|}{$\geq 6.9$ group } \\
\hline & & Total & $\begin{array}{c}\text { DM } \\
\text { control } \\
\text { group }\end{array}$ & $\begin{array}{l}\text { Poor } \\
\text { control } \\
\text { group }\end{array}$ & & Total & $\begin{array}{c}\text { DM } \\
\text { control } \\
\text { group }\end{array}$ & $\begin{array}{l}\text { Poor } \\
\text { control } \\
\text { group }\end{array}$ \\
\hline $\mathrm{N}$ & 212 & 334 & 182 & 152 & 187 & 273 & 153 & 120 \\
\hline Ca blocker (\%) & 44.8 & 44.9 & 47.3 & 42.1 & 46.6 & 47.9 & 48.3 & 47.4 \\
\hline ACEI (\%) & 7.5 & 10.5 & 9.3 & 11.8 & 7.3 & 11.4 & 11.5 & 11.2 \\
\hline Statin (\%) & 68.9 & 74.6 & 81.9 & $65.8^{\ddagger}$ & 76.6 & 73.1 & 80.8 & $63.8^{\ddagger}$ \\
\hline ARB (\%) & 69.3 & 71.0 & 76.9 & $63.8^{\ddagger}$ & 71.4 & 69.5 & 75.3 & $62.5^{\dagger \dagger}$ \\
\hline Sulfonylurea (\%) & 23.1 & 25.4 & 22.5 & 28.9 & 20.4 & $28.7^{*}$ & 25.3 & 32.9 \\
\hline$\alpha \mathrm{GI}(\%)$ & 17.9 & 21.6 & 21.4 & 21.7 & 18.5 & 22.5 & 19.8 & 25.7 \\
\hline Thiazolidine (\%) & 3.8 & $9.9^{* *}$ & 9.3 & 10.5 & 4.9 & $12.6^{\star *}$ & 11.0 & 14.5 \\
\hline Insulin (\%) & 18.9 & $45.8^{\dagger}$ & 48.4 & 42.8 & 12.6 & $32.9^{\dagger}$ & 33.0 & 32.9 \\
\hline Biguanides (\%) & 7.1 & 8.1 & 7.1 & 9.2 & 8.7 & 9.9 & 11.0 & 8.6 \\
\hline
\end{tabular}

$<6.9$ group vs. $\geq 6.9$ group: $P$-value $*<0.05,{ }^{* *}<0.01,{ }^{\dagger}<0.001$.

DM control group vs. Poor control group: $P$-value ${ }^{t \dagger}<0.05, \neq<0.01$.

$\mathrm{PCl}$, percutaneous coronary intervention; ACEI, angiotensin converting enzyme inhibitor; ARB, angiotensin receptor blocker; $\alpha \mathrm{Gl}, \alpha$-glucosidase inhibitor.

$\mathrm{HbA}_{1 \mathrm{c}}$ values: we used the National Glycohemoglobin Standardization Program (NGSP) ${ }^{15}$ value.

\begin{tabular}{|c|c|c|c|c|}
\hline & \multirow[b]{2}{*}{$<6.9$ group } & \multicolumn{3}{|c|}{$\geq 6.9$ group } \\
\hline & & Total & $\begin{array}{l}\text { DM control } \\
\text { group }\end{array}$ & $\begin{array}{l}\text { Poor control } \\
\text { group }\end{array}$ \\
\hline N & 246 & 374 & 208 & 166 \\
\hline RCA/LAD/Cx (\%) & $78 / 124 / 44$ & $129 / 168 / 77$ & $72 / 92 / 44$ & $57 / 76 / 33$ \\
\hline Pre-ISR (\%) & 19.5 & 23.8 & 24.5 & 22.9 \\
\hline AHA/ACC type B2+C (\%) & 72.8 & 70.3 & 68.8 & 72.3 \\
\hline Severe Calcification (\%) & 14.2 & 10.4 & 10.6 & 10.2 \\
\hline DES (\%) & 48.0 & 54.8 & 59.1 & 49.4 \\
\hline \multicolumn{5}{|l|}{ QCA } \\
\hline \multicolumn{5}{|l|}{ Pre-procedural results } \\
\hline $\mathrm{N}$ & 128 & 255 & 142 & 113 \\
\hline Lesion length (mm) & $18.5 \pm 9.9$ & $18.9 \pm 11.4$ & $19.5 \pm 12.1$ & $18.1 \pm 10.4$ \\
\hline Reference $(\mathrm{mm})$ & $2.6 \pm 0.6$ & $2.5 \pm 0.6$ & $2.5 \pm 0.7$ & $2.5 \pm 0.6$ \\
\hline $\operatorname{MLD}(\mathrm{mm})$ & $0.85 \pm 0.41$ & $0.74 \pm 0.41^{\star \star}$ & $0.73 \pm 0.43$ & $0.74 \pm 0.38$ \\
\hline$\% D S(\%)$ & 68.5 & $71.7^{\star}$ & 71.5 & 72 \\
\hline \multicolumn{5}{|l|}{ Post-procedural results } \\
\hline $\operatorname{MLD}(\mathrm{mm})$ & $1.96 \pm 0.54$ & $1.90 \pm 0.59$ & $1.90 \pm 0.62$ & $1.90 \pm 0.54$ \\
\hline$\% D S(\%)$ & 26.3 & 27.3 & 27.2 & 27.4 \\
\hline Stent length $(\mathrm{mm})$ & $23.1 \pm 11.4$ & $24.3 \pm 12.7$ & $24.5 \pm 14.3$ & $24.0 \pm 10.0$ \\
\hline \multicolumn{5}{|l|}{ IVUS results } \\
\hline $\mathrm{N}$ & 91 & 174 & 98 & 75 \\
\hline Lesion EEM CSA $\left(\mathrm{mm}^{2}\right)$ & $13.8 \pm 4.5$ & $14.1 \pm 4.9$ & $13.7 \pm 5.0$ & $14.8 \pm 4.7$ \\
\hline Lesion lumen CSA $\left(\mathrm{mm}^{2}\right)$ & $6.6 \pm 2.3$ & $6.4 \pm 2.4$ & $6.2 \pm 2.3$ & $6.5 \pm 2.5$ \\
\hline Lesion atheroma CSA $\left(\mathrm{mm}^{2}\right)$ & $7.3 \pm 2.8$ & $7.8 \pm 3.3$ & $7.4 \pm 3.4$ & $8.2 \pm 3.1$ \\
\hline Lesion \% plaque (\%) & 51.7 & $54.3^{*}$ & 53.2 & 55.6 \\
\hline Minimum stent CSA $\left(\mathrm{mm}^{2}\right)$ & $6.6 \pm 2.4$ & $6.4 \pm 2.4$ & $6.3 \pm 2.3$ & $6.6 \pm 2.5$ \\
\hline
\end{tabular}

$<6.9$ group vs. $\geq 6.9$ group: $P$-value ${ }^{*}<0.05,{ }^{* *}<0.01$

QCA, quantitative coronary angiography; IVUS, intravascular ultrasound; ISR, instent restenosis; DES, drug eluting stent; MLD, minimum lumen diameter; \%DS, percent diameter stenosis; EEM, external elastic membrane; CSA, cross sectional area. Other abbreviation see in Table 1.

$\mathrm{HbA}_{1 \mathrm{c}}$ values: we used the National Glycohemoglobin Standardization Program (NGSP) ${ }^{15}$ value. 


\begin{tabular}{|c|c|c|c|c|}
\hline & \multirow[b]{2}{*}{$<6.9$ group } & \multicolumn{3}{|c|}{$\geq 6.9$ group } \\
\hline & & Total & $\begin{array}{l}\text { DM control } \\
\text { group }\end{array}$ & $\begin{array}{c}\text { Poor control } \\
\text { group }\end{array}$ \\
\hline $\mathrm{N}$ & 187 & 273 & 153 & 120 \\
\hline ISR (\%) & 25.1 & $31.5^{\star}$ & 34.0 & 28.3 \\
\hline Follow up QCA results, $\mathrm{N}$ & 109 & 199 & 110 & 89 \\
\hline Lesion MLD (mm) & $1.65 \pm 0.66$ & $1.58 \pm 0.74$ & $1.57 \pm 0.74$ & $1.60 \pm 0.74$ \\
\hline Lesion \%DS (\%) & 38.9 & 40.6 & 40.7 & 40.6 \\
\hline Stent \%DS (\%) & 28.5 & 25.9 & 28.3 & 22.5 \\
\hline Lesion late loss (mm) & $0.23 \pm 0.66$ & $0.31 \pm 0.71$ & $0.36 \pm 0.71$ & $0.25 \pm 0.70$ \\
\hline Stent late-loss (mm) & $0.37 \pm 0.76$ & $0.38 \pm 0.66$ & $0.47 \pm 0.71$ & $0.26 \pm 0.55^{t \dagger}$ \\
\hline
\end{tabular}

$<6.9$ group vs. $\geq 6.9$ group: $P$-value * $<0.05$.

DM control group vs. Poor control group: $\mathrm{P}$-value ${ }^{\dagger+}<0.05$.

Abbreviations see in Tables 1,3.

$\mathrm{HbA}_{1 c}$ values: we used the National Glycohemoglobin Standardization Program (NGSP) ${ }^{15}$ value.

group: mean Pre-HbAic was $6.3 \pm 0.4 \%)]$ and the poor-glycemic-control group [Pre-HbAic values greater than $6.9 \%(\geq 6.9$ group: mean Pre-HbAlc was $8.2 \pm 1.2 \%$ )], while the median value of $\mathrm{HbA}_{1 \mathrm{c}}$ in whole diabetic patients were $7.2 \%$. In the $\geq 6.9$ group, there was a greater incidence of a history of coronary artery bypass graft $(\mathrm{CABG})(7.8 \%$ vs. $1.9 \%, \mathrm{P}<0.01)$ or 3 -vessel disease $(23.7 \%$ vs. $15.6 \%, \mathrm{P}<0.05)$, and a lower incidence of complicating hypertension (HT) $(69.8 \%$ vs. $78.3 \%$, $\mathrm{P}<0.05)$, compared to those in the $<6.9$ group. Blood tests showed lower concentrations of creatinine $(1.0 \pm 0.5 \mathrm{mg} / \mathrm{dl}$ vs. $1.1 \pm 0.6 \mathrm{mg} / \mathrm{dl}, \mathrm{P}<0.01)$ and higher total cholesterol (TC) concentrations $(189.1 \pm 37.6 \mathrm{mg} / \mathrm{dl}$ vs. $179.3 \pm 42.0 \mathrm{mg} / \mathrm{dl}, \mathrm{P}<0.05)$ in the $\geq 6.9$ group. In the $\geq 6.9$ group, the mean age was greater $(67.9 \pm 9.4$ vs. $65.8 \pm 9.1, \mathrm{P}<0.05)$, but Pre-HbA1c $(7.9 \pm 1.0 \%$ vs. $8.4 \pm 1.3 \%, \mathrm{P}<0.001)$ and the frequencies of HT $(62.5 \%$ vs. $75.8 \%, \mathrm{P}<0.01$ ) were lower in the Poor control group than those in the DM control group.

With regard to the intake of medications at PCI (Table 2), while no differences were observed in antihypertensive agents or statins between the $<6.9$ group and $\geq 6.9$ group, the usage rates of thiazolidine and insulin were significantly higher in the $\geq 6.9$ group than in the $<6.9$ group (thiazolidine usage rate: $9.9 \%$ vs. $3.8 \%, \mathrm{P}<0.01$; insulin usage rate: $45.8 \%$ vs. $18.9 \%, \mathrm{P}<0.001$, respectively). Among the $\geq 6.9$ group, the DM control group showed a higher rate of statin $(\mathrm{P}<0.001)$ and angiotensin II receptor blockers $(\mathrm{ARB})(\mathrm{P}<0.01)$ usage than the Poor control group. These patterns were maintained after the follow-up periods.

Although both groups had similar lesion characteristics (Table 3), the $<6.9$ group had a greater MLD $(0.85 \pm 0.41 \mathrm{~mm}$ vs. $0.74 \pm 0.41 \mathrm{~mm}, \mathrm{P}<0.01)$ and a smaller $\%$ diameter stenosis (\%DS) $(68.5 \%$ vs. $71.7 \%, \mathrm{P}<0.05)$ than those in the $\geq 6.9$ group (pre-procedural results). Among post-procedural results, no difference was observed between the 2 groups except that the $\%$ plaque area on IVUS in the $<6.9$ group $(51.7 \%$ vs. $54.3 \%, \mathrm{P}<0.05)$ was smaller than that in the $\geq 6.9$ group. No significance was observed between the DM control group vs. the Poor control group.

Follow-up angiographic results (Table 4) showed no differences in MLD, \%DS, late loss, etc, except that the in-stent restenosis rate (ISR) in the $<6.9$ group was lower than that in the $\geq 6.9$ group ( $25.1 \%$ vs. $31.5 \%, \mathrm{P}<0.05$, respectively). Among the $\geq 6.9$ group, the DM control group showed greater stent late loss than in the Poor control group $(0.47 \pm 0.71 \mathrm{~mm}$ vs. $0.26 \pm 0.55 \mathrm{~mm}, \mathrm{P}<0.05)$.

With regard to follow-up clinical outcomes (Table 5), while no differences were observed in death or MI, the incidence of MACE in the $<6.9$ group was significantly lower than that in the $\geq 6.9$ group ( $18.4 \%$ vs. $26.2 \%, \mathrm{P}<0.05)$. TLRPCI was lower in the $<6.9$ group than in the $\geq 6.9$ group (16.2\% vs. $22.7 \%, \mathrm{P}<0.05)$. Follow-up $\mathrm{HbA}_{1 \mathrm{c}}$ in the $<6.9$ group was smaller than that in the $\geq 6.9$ group $(6.6 \pm 1.0 \%$ vs. $7.7 \pm 1.3 \%, \mathrm{P}<0.001$, respectively). Among the $\geq 6.9$ group, follow-up $\mathrm{HbA}_{1 c}$ in the DM control group was smaller than that in the Poor control group $(7.3 \pm 1.0 \%$ vs. $8.5 \pm 1.3 \%$, $\mathrm{P}<0.001$, respectively). The $\mathrm{HbA}_{1 c}$ difference (Pre-HbAlcFollow-up $\mathrm{HbA}_{1 c}$ ) in the DM control group was $1.1 \pm 1.1 \%$, while an increase, and thus a negative value for the $\mathrm{HbAlc}_{1 \mathrm{c}}$ difference $(-0.7 \pm 0.8 \%)$, was observed in the Poor control group $(\mathrm{P}<0.001)$.

We performed a multivariate analysis to investigate if the Pre-HbA 1 , Pre-HbA $\mathrm{H}_{1 \mathrm{c}} \geq 6.9 \%$ or $\mathrm{HbA}_{1 c}$ difference was associated with the occurrence of MACE. In this analysis, we considered parameters for which there were significant intergroup differences (Table 6). For the entire DM group, a multivariate analysis was performed with the following models: Model 1: Single Pre-HbAlc, Model 2: Single Pre$\mathrm{HbA}_{1 \mathrm{c}} \geq 6.9 \%$, Model 3: Single HbA1c difference, Model 4: Pre-HbA $1 c+$ Pre-HbA $1 c \geq 6.9 \%+\mathrm{HbA}_{1 c}$ difference, Model 5: Model 4+HT, dyslipidemia (DL), DES and TC (from patient background parameters), Model 6: Model 5+MLD, \%DS, lesion length and lesion reference (from QCA and pre-procedural results parameters), Model 7: Model 6+statin, ARB, insulin and thiazolidine (from medications at PCI). As a result, MLD and \%DS were associated with MACE in every model used in the analysis (Model 6=MLD: odds ratio $[\mathrm{OR}]=5.54,95 \%$ confidence interval [CI] [1.15-31.17], $\% \mathrm{DS}$ : $\mathrm{OR}=1.10,95 \% \mathrm{CI}$ [1.07-1.14]) (data not tabulated). However, no relationship was observed in any of these models between the occurrence of MACE and either Pre-HbAic alone, Pre-HbA1c $\left(6.9 \%\right.$ or $\mathrm{HbA}_{1 \mathrm{c}}$ difference in Model 3 to 7.

In the entire $\geq 6.9$ group, a multivariate analysis was performed with each of the following models: Model 1: Single Pre-HbA1c, Model 3: Single HbA1c difference, Model 4: Pre$\mathrm{HbA}_{1 \mathrm{c}}+\mathrm{HbA}_{1 \mathrm{c}}$ difference, Model 5: Model 4+Age, HT, DL, DES and pre-instent restenosis (from patient background parameters), Model 6: Model 5+lesion reference (from QCA and pre-procedural results parameters) + stent length (from post-procedural results), Model 7: Model 6+statin, ARB, insulin, thiazolidine (from medications at PCI). Pre-instent restenosis and lesion reference were both associated with MACE in each of the models used in the analysis (Model 


\begin{tabular}{|c|c|c|c|c|}
\hline & \multirow[b]{2}{*}{$<6.9$ group } & \multicolumn{3}{|c|}{$\geq 6.9$ group } \\
\hline & & Total & $\begin{array}{l}\text { DM control } \\
\text { group }\end{array}$ & $\begin{array}{l}\text { Poor control } \\
\text { group }\end{array}$ \\
\hline $\mathbf{N}$ & 185 & 321 & 176 & 145 \\
\hline Follow-up days (day) & $326.0 \pm 380.8$ & $288.9 \pm 284.5$ & $287.1 \pm 275.9$ & $291.1 \pm 295.5$ \\
\hline BMI $\left(\mathbf{k g} / \mathbf{m}^{2}\right)$ & $24.3 \pm 3.9$ & $24.3 \pm 3.9$ & $24.1 \pm 3.6$ & $24.6 \pm 4.3$ \\
\hline \multicolumn{5}{|c|}{ Follow-up blood sampling test } \\
\hline $\mathrm{HbA}_{1 \mathrm{c}}(\%)$ & $6.6 \pm 1.0$ & $7.7 \pm 1.3^{\dagger}$ & $7.3 \pm 1.0$ & $8.5 \pm 1.3^{\ddagger \ddagger}$ \\
\hline $\mathrm{HbA}_{1 c}$ difference $(\%)$ & $-0.3 \pm 0.8$ & $0.4 \pm 1.3$ & $1.1 \pm 1.1$ & $-0.7 \pm 0.8 \neq \ddagger$ \\
\hline \multicolumn{5}{|c|}{ Follow-up clinical outcomes } \\
\hline MACE (\%) & 18.4 & $26.2^{*}$ & 27.3 & 24.8 \\
\hline LST (\%) & 2.7 & 1.9 & 2.3 & 1.4 \\
\hline Death $(\%)$ & 1.1 & 2.2 & 1.7 & 2.8 \\
\hline $\mathrm{Ml}(\%)$ & 2.7 & 2.2 & 2.8 & 1.4 \\
\hline TLR-PCI (\%) & 16.2 & $22.7^{*}$ & 23.9 & 21.4 \\
\hline TLR-CABG (\%) & 0.5 & 0 & 0 & 0 \\
\hline NTV-PCI (\%) & 12.4 & 13.4 & 13.1 & 13.8 \\
\hline NTV-CABG(\%) & 0.5 & 0 & 0 & 0 \\
\hline
\end{tabular}

$<6.9$ group vs. $\geq 6.9$ group: $P$-value * $<0.05, t<0.001$.

DM control group vs. Poor control group: P-value $¥ \ddagger<0.001$.

$\mathrm{Hb} A_{1 c}$ difference, Pre-HbA $1 c-\mathrm{HbA}_{1 c}$ at follow-up; MACE, major adverse cardiovascular events; LST, late stent thrombosis; TLR, target lesion revascularization; NTV, non-target vessel. Other abbreviations see in Tables 1,2.

$\mathrm{HbA}_{1 \mathrm{c}}$ values: we used the National Glycohemoglobin Standardization Program (NGSP) ${ }^{15}$ value.

Table 6. Multivariate Logistic Regression Analysis: $\mathrm{HbA}_{1 \mathrm{c}}$ and MACE

\begin{tabular}{|c|c|c|c|c|c|c|c|}
\hline \multirow{3}{*}{ Whole DM group } & \multicolumn{7}{|c|}{ Odds ratio ( $95 \%$ confidence interval) } \\
\hline & Model 1 & Model 2 & Model 3 & Model 4 & Model 5 & Model 6 & Model 7 \\
\hline & & & & & & & \\
\hline Pre-HbA & $\begin{array}{c}1.15 \\
(0.98-1.33)\end{array}$ & & & $\begin{array}{c}0.95 \\
(0.74-1.22)\end{array}$ & $\begin{array}{c}0.95 \\
(0.73-1.21)\end{array}$ & $\begin{array}{c}0.88 \\
(0.58-1.34)\end{array}$ & $\begin{array}{c}0.85 \\
(0.53-1.35)\end{array}$ \\
\hline Pre-HbA $1 \mathrm{c}(\geq 6.9 \%)$ & & $\begin{array}{c}1.55 \\
(1.02-2.36)^{\star}\end{array}$ & & $\begin{array}{c}1.57 \\
(0.83-3.06)\end{array}$ & $\begin{array}{c}1.54 \\
(0.79-3.06)\end{array}$ & $\begin{array}{c}1.52 \\
(0.46-5.10)\end{array}$ & $\begin{array}{c}1.19 \\
(0.32-4.50)\end{array}$ \\
\hline $\mathrm{Hb} \mathrm{A}_{1 \mathrm{c}}$ difference & & & $\begin{array}{c}1.12 \\
(0.93-1.34)\end{array}$ & $\begin{array}{c}1.10 \\
(0.89-1.38)\end{array}$ & $\begin{array}{c}1.14 \\
(0.91-1.44)\end{array}$ & $\begin{array}{c}0.79 \\
(0.52-1.26)\end{array}$ & $\begin{array}{c}0.80 \\
(0.51-1.28)\end{array}$ \\
\hline$\geq 6.9$ group & & & & & & & \\
\hline Pre-HbA $1 c$ & $\begin{array}{c}1.07 \\
(0.86-1.30)\end{array}$ & - & & $\begin{array}{c}0.94 \\
(0.72-1.21)\end{array}$ & $\begin{array}{c}0.94 \\
(0.69-1.25)\end{array}$ & $\begin{array}{c}1.02 \\
(0.69-2.47)\end{array}$ & $\begin{array}{c}1.02 \\
(0.67-1.55)\end{array}$ \\
\hline $\mathrm{HbA}_{1 c}$ difference & & & $\begin{array}{c}1.10 \\
(0.90-1.34)\end{array}$ & $\begin{array}{c}1.14 \\
(0.90-1.45)\end{array}$ & $\begin{array}{c}1.22 \\
(0.92-1.66)\end{array}$ & $\begin{array}{c}1.25 \\
(0.87-1.88)\end{array}$ & $\begin{array}{c}1.17 \\
(0.79-1.81)\end{array}$ \\
\hline
\end{tabular}

$P$-value * $<0.05$.

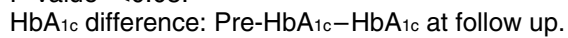

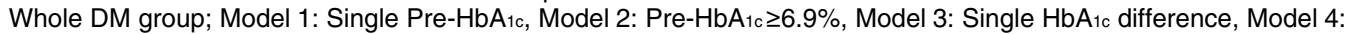

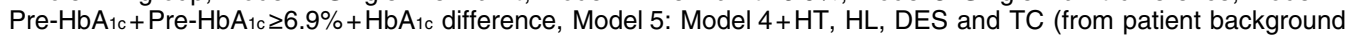
parameters), Model 6: Model 5+MLD, \%DS, lesion length and lesion reference (from QCA and pre-procedural results parameters), Model 7: Model 6+statin, ARB, insulin and thiazolidine (from medications at PCl).

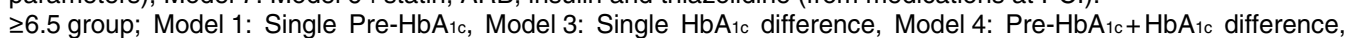

Model 5: Model 4+Age, HT, HL, DES and pre-instent restenosis (from patient background parameters), Model 6: Model 5 +lesion reference (from QCA and pre-procedural results parameters)+stent length (from post-procedural results), Model 7: Model $6+$ statin, $A R B$, insulin, thiazolidine (from medications at $\mathrm{PCl}$ ).

Abbreviations see in Tables 1-3,5.

$\mathrm{HbA}_{1 c}$ values: we used the National Glycohemoglobin Standardization Program (NGSP) ${ }^{15}$ value.

6=pre-instent restenosis: $\mathrm{OR}=3.50$ [1.23-9.98], lesion reference: $\mathrm{OR}=0.44$ [0.17-1.00]) (data not tabulated). However, no relationship was observed in any of the models between MACE and Pre-HbAic or the HbAlc difference.

\section{Discussion}

In Japan, the number of DM patients has been increasing rapidly, and DM is one of the major risk factors of CVD. ${ }^{20,21}$ Although it has been reported that $70-80 \%$ of the prognosis of DM patients is determined by $\mathrm{CVD},{ }^{22}$ we have not yet been able to obtain satisfactory outcomes by means of revascularization. A high incidence of stent restenosis is often observed in DM patients. In our database of the FU-Registry, as in other reports, in a comparison of subjects who developed stent restenosis ( $\mathrm{n}=231$ lesions) to those who did not $(\mathrm{n}=682$ lesions), a multivariate analysis revealed that DM was associated with stent restenosis $(\mathrm{OR}=1.79)$, along with other factors such as stent length and lesion reference (unpublished data). Since the occurrence or non-occurrence 
of MACE is a major prognostic determinant for the post-PCI life of DM patients, ${ }^{3,23}$ we investigated the impact of glycemic control on the clinical outcome in DM with PCI.

Corpus et al studied the rate of vascular restenosis in patients with $\mathrm{HbA}_{1 \mathrm{c}}$ values above $7.0 \%$ at the time of PCI and in patients who underwent intensive glycemic control and had $\mathrm{HbA}_{1 \mathrm{c}}$ values of $<7.0 \%$ or below at the time of PCI. ${ }^{12}$ They found a significantly lower incidence of TLRPCI in the $\mathrm{HbA}_{1 \mathrm{c}}<7.0 \%$ group (15\% vs. $34 \%$ ), and reported that Pre-HbAlc values could be recognized as an independent predictor of stent restenosis. They suggested that intensive glycemic control could improve the clinical outcome in patients undergoing PCI. However, because they measured $\mathrm{HbA}_{1 c}$ only at the time of PCI and made no reference to post-PCI glycemic control, the favorable follow-up clinical outcomes might be considered to be due to intensive glycemic control before PCI.

In our comparison of the follow-up clinical outcomes in the Pre-HbA $1 c<6.9$ and Pre-HbA $1 c \geq 6.9$ groups, the $<6.9$ group showed significantly lower incidences of ISR and TLR, and consequently showed more favorable outcomes with a lower prevalence of MACE. To exclude the possible effect of DES, ${ }^{12}$ although no significant difference was observed in the usage rate of DES between the 2 groups, we performed a comparative analysis that only considered patients with a BMS, ie, the $<6.9$ group showed significantly lower incidences of ISR ( $33.1 \%$ vs. $43.3 \%, \mathrm{P}<0.05)$, MACE $(23.1 \%$ vs. $36.2 \%, \mathrm{P}<0.05)$ and TLR-PCI $(19.7 \%$ vs. $31.9 \%, \mathrm{P}<0.05)$ than the (6.9 groups (data not tabulated), which was similar to the results as a whole (BMS+DES) (Tables 4 and 5).

We can speculate regarding why such favorable clinical results were seen in the $<6.9$ group compared to the $\geq 6.9$ group even though Pre-HbAlc and Pre-HbAlc (6.9\% were not related to MACE by a multivariate analysis (Table 6). Regarding the usage and intake rates of oral hypoglycemic agents, more patients in the $<6.9$ group were believed to have remained under relatively good glycemic control since the pre-PCI period. Therefore, patients in the $<6.9$ group were thought to have been affected less by factors that arose from chronic hyperglycemia and an increasing incidence of MACE. However, because the $\geq 6.9$ group showed some unfavorable lesion characteristics such as a smaller MLD and higher \% DS by QCA, or a higher lesion percent plaque in the IVUS post-procedural results, we could not exclude the possibility that the morphological disadvantage associated with diabetes as well as the duration of the illness ${ }^{24}$ might have influenced the follow-up clinical outcome.

As a potential explanation for the similar follow-up clinical outcomes seen in the DM control and Poor control groups, we speculated that the 'metabolic memory (Legacy)' effects referred to in the DCCT/EDIC ${ }^{13,14}$ and UKPDS80 ${ }^{1}$ studies, etc., might have affected the study results. Despite mild to moderate glycemic control before and after PCI, due to metabolic memory (Legacy) effects, some MACE-increasing factors, which are due to chronic hyperglycemia, might have adversely affected the clinical outcome equally in both of the $\geq 6.9$ groups. These factors might have included vascular endothelial dysfunction, an activated blood-clotting system, and several types of inflammatory cytokines. Our findings suggest that, to prevent MACE, it is essential that good glycemic control should always be maintained before PCI so that when PCI is performed, the vessels should be in improved and normalized conditions with reduced MACEincreasing factors as mentioned above. Our findings also suggest that post-PCI glycemic control or post-PCI changes

\begin{tabular}{|lcc|}
\hline Table 7. DM Control Group vs. Poor Control Group in $<6.9$ \\
Group
\end{tabular}

DM control group vs. Poor control group: P-value $\$ \neq<0.001$.

Abbreviations see in Tables 1-3,5.

$\mathrm{HbA}_{1 \mathrm{c}}$ values: we used the National Glycohemoglobin Standardization Program (NGSP) ${ }^{15}$ value.

in $\mathrm{HbA}_{1 \mathrm{c}}$ values might not be related to the improvement of the intermediate follow-up clinical outcome, including TLR. However, these data do not support the concept that DM control should not be advised after PCI, because a life-style change might be essential for diabetic patients. ${ }^{25}$

Further, we divided the $<6.9$ group into 2 groups and compared their clinical outcomes. One group consisted of patients who had lower $\mathrm{HbA}_{1 \mathrm{c}}$ values at follow-up, and the other consisted of those who had the same or increased $\mathrm{HbAlc}_{1 \mathrm{c}}$ values at follow-up. In the former group, the $\mathrm{HbA}_{1 c}$ difference was $0.4 \pm 0.2 \%$, and in the latter this value was $-0.6 \pm 0.8 \%$. Nevertheless, the follow-up clinical outcomes in the 2 groups were similar (ISR: $26.3 \%$ vs. $24.8 \%, \mathrm{P}=0.81$, MACE: $12.2 \%$ vs. $20.1 \%$, $\mathrm{P}=0.26$, MI: $2.4 \%$ vs. $2.8 \%, \mathrm{P}=0.69$, TLR: $12.2 \%$ vs. $17.4 \% ; \mathrm{P}=0.27$, respectively) (Table 7). Both groups had more favorable clinical outcomes than the $\geq 6.9$ group. The results of our analysis suggest that follow-up clinical outcomes are not influenced by post-PCI changes in $\mathrm{HbAlc}_{1 \mathrm{val}}$ ues, and that the application of good glycemic control continuously from the pre-PCI period is associated with an improved clinical outcome at follow-up.

A greater incidence of a history of CABG or three-vessel disease was observed in our study in the $\geq 6.9$ group (Table 1 ), and similar data were recently reported by Yamagata et al. ${ }^{26}$ $\mathrm{CAD}$ in diabetes is associated with several characteristics, including small vessel size, more diffuse, calcifications, etc. Our data in DM patients focused on the target lesions, rather than on the different kinds of diabetic $\mathrm{CAD},{ }^{27}$ and further trials are needed.

Since the lifestyle of the mean 67-year-old Japanese participants in this study were presumed to be quite different from 
from those in western countries, Japanese evidence should be reported. We categorized patients into $\mathrm{HbA}_{1 \mathrm{c}}<6.9$ and $\geq 6.9$ groups, and these $\mathrm{HbA}_{1 \mathrm{c}}$ values adjusted by NGSP were similar to the 1995 ACC/AHA secondary prevention guideline ${ }^{28}$ of $\mathrm{HbA}_{1 \mathrm{c}}<7.0 \%$, and to the risk factor goals $(\mathrm{HbA} 1 \mathrm{c}$ $<7.0 \%$ ) in other clinical trials, such as the COURAGE Trials, ${ }^{29}$ etc. Thus, the practical guidelines in Japan are becoming very similar to those in western countries despite the difference in lifestyle patterns. Again, this is the first paper dealing with Japanese in southern Japan to show the importance of glycemic control in the long-term clinical outcome of PCI.

\section{Study Limitations}

This study consisted of an analysis of patients who underwent PCI from 2003 to 2008, and was not a prospective cohort study. A cohort study might further verify our findings. Although we performed QCA and IVUS in randomly selected participants (QCA in about $45 \%$ and IVUS in onethird of the subjects), it would be useful for all of the subjects to undergo these examinations so that differences between the groups might be more recognizable. Also, by obtaining laboratory data regarding various cytokines, or lipoprotein subfractions of negatively-charged low-density lipoprotein cholesterol, ${ }^{30}$ as we previously reported, or by collecting detailed data regarding pre-PCI glycemic control, history of DM etc, we might be able to verify our study results, and this could help us to solve new problems such as, 'Do mean prePCI HbAic values have any relationship with MACE?' and 'How long should a patient with DM continue good glycemic control before PCI to achieve nearly the same clinical outcome as a patient without DM?' In the present study, we did not analyze the doses of the drugs administered. Therefore, we cannot rule out the possibility that there might have been a difference in the doses administered. No difference was observed regarding the use of hypoglycemic agents between the DM control group and Poor control group. Therefore, we cannot exclude the possibility that the clinical outcome might have been affected for the above reasons.

\section{Conclusions}

The clinical outcomes in the $<6.9$ group were significantly better than those in the $\geq 6.9$ group. However, no relationship was observed between the incidence of MACE and Pre$\mathrm{HbA}_{1 c}$ or Pre-HbAlc $\geq 6.9 \%$, which suggests that pre-PCI glycemic control affects lesion characteristics. In patients with DM, the present results suggest that intensive glycemic control started at PCI and continued through the follow-up period was not associated with an improved clinical outcome at follow-up.

\section{Disclosures}

This work was supported by a grant-in-aid from the Ministry of Education, Science and Culture of Japan (No. 21590960), and by research grants from the Central Research Institute of Fukuoka University (2005-2009) and the FU-Global program (2008-2010).

\section{References}

1. Holman RR, Paul SK, Bethel MA, Matthews DR, Neil HA. 10year follow-up of intensive glucose control in type 2 diabetes. $N$ Engl J Med 2008; 359: 1577-1589.

2. Garcia MJ, McNamara PM, Gordon T, Kannel WB. Morbidity and mortality in diabetics in the Framingham population: Sixteen year follow-up study. Diabetes 1974; 23: 105-111.

3. Flaherty JD, Davidson CJ. Diabetes and coronary revasculariza- tion. JAMA 2005; 293: 1501-1508

4. Cutlip DE, Chhabra AG, Baim DS, Chauhan MS, Marulkar S, Massaro J, et al. Beyond restenosis: Five-year clinical outcomes from second-generation coronary stent trials. Circulation 2004; 110: $1226-1230$.

5. Lee TT, Feinberg L, Baim DS, Holmes DR, Aroesty JM, Carrozza JP Jr, et al. Effect of diabetes mellitus on five-year clinical outcomes after single-vessel coronary stenting (a pooled analysis of coronary stent clinical trials). Am J Cardiol 2006; 98: 718-721.

6. Nakatani D, Sakata Y, Mizuno H, Shimizu M, Suna S, Usami M, et al; Osaka Acute Coronary Insufficiency Study (OACIS) Group. Impact of diabetes mellitus on rehospitalization for heart failure among survivors of acute myocardial infarction in the percutaneous coronary intervention era. Circ J 2009; 73: 662-666.

7. Stehouwer CD, Lambert J, Donker AJ, van Hinsbergh VW. Endothelial dysfunction and pathogenesis of diabetic angiopathy. Cardiovasc Res 1997; 34: 55-68.

8. Farah R, Shurtz-Swirski R, Lapin O. Intensification of oxidative stress and inflammation in type 2 diabetes despite antihyperglycemic treatment. Cardiovasc Diabetol 2008; 7: 20.

9. Tchaikovski V, Olieslagers S, Bohmer FD, Waltenberger J. Diabetes mellitus activates signal transduction pathways resulting in vascular endothelial growth factor resistance of human monocytes. Circulation 2009; 120: 150-159.

10. McBane RD 2nd, Hardison RM, Sobel BE; BARI 2D Study Group. Comparison of plasminogen activator inhibitor-1, tissue type plasminogen activator antigen, fibrinogen, and D-dimer levels in various age decades in patients with type 2 diabetes mellitus and stable coronary artery disease (from the BARI 2D trial). Am J Cardiol 2010; 105: 17-24.

11. Airoldi F, Briguori C, Iakovou I, Stankovic G, Biondi-Zoccai G, Carlino M, et al. Comparison of sirolimus versus paclitaxel eluting stents for treatment of coronary in-stent restenosis. Am J Cardiol 2006; 97: $1182-1187$

12. Corpus RA, George PB, House JA, Dixon SR, Ajluni SC, Devlin $\mathrm{WH}$, et al. Optimal glycemic control is associated with a lower rate of target vessel revascularization in treated type II diabetic patients undergoing elective percutaneous coronary intervention. J Am Coll Cardiol 2004; 43: 8-14.

13. Writing Team for the Diabetes Control and Complications Trial/ Epidemiology of Diabetes Interventions and Complications Research Group. Effect of intensive therapy on the microvascular complications of type 1 diabetes mellitus. JAMA 2002; 287: 2563-2569.

14. Writing Team for the Diabetes Control and Complications Trial/ Epidemiology of Diabetes Interventions and Complications Research Group. Sustained effect of intensive treatment of type 1 diabetes mellitus on development and progression of diabetic nephropathy: The Epidemiology of Diabetes Interventions and Complications (EDIC) study. JAMA 2003; 290: 2159-2167.

15. Japan Diabetes Society Report. http://www.jds.or.jp/jds_or_jp0/ uploads/photos/636.pdfl (accessed 10 August, 2010).

16. Guidelines for the primary prevention of ischemic heart disease revised version (JCS 2006). http://www.j-circ.or.jp/guideline/pdf/ JCS2006_kitabatake_h.pdf (accessed 10 August, 2010).

17. Fukuda Y, Shirai K, Miura S, Ike A, Takamiya Y, Kuwano T, et al. The impact of angulated lesions on angiographic late loss in patients with drug-eluting stent implantation. J Cardiol 2009; 53: 396401.

18. Yanagi D, Shirai K, Takamiya Y, Fukuda Y, Kuwano T, Ike A, et al. Results of provisional stenting with a Sirolimus-eluting stent for bifurcation lesion: Multicenter study in Japan. J Cardiol 2008; 51: 89-94.

19. Iwata A, Miura S, Shirai K, Kawamura A, Tomita S, Matsuo Y, et al. Lower level of low-density lipoprotein cholesterol by statin prevents progression of coronary restenosis after successful stenting in acute myocardial infarction. Intern Med 2006; 45: 885-890.

20. Mukai N, Doi Y, Ninomiya T, Hata J, Yonemoto K, Iwase M, et al. Impact of metabolic syndrome compared with impaired fasting glucose on the development of type 2 diabetes in a general Japanese population: The Hisayama study. Diabetes Care 2009; 32: $2288-$ 2293.

21. Osawa H, Doi Y, Makino H, Ninomiya T, Yonemoto K, Kawamura $\mathrm{R}$, et al. Diabetes and hypertension markedly increased the risk of ischemic stroke associated with high serum resistin concentration in a general Japanese population: The Hisayama Study. Cardiovasc Diabetol 2009; 8: 60 .

22. Moreno PR, Fuster V. New aspects in the pathogenesis of diabetic atherothrombosis. J Am Coll Cardiol 2004; 44: 2293-2300.

23. Nicholls SJ, Tuzcu EM, Kalidindi S, Wolski K, Moon KW, Sipahi $\mathrm{I}$, et al. Effect of diabetes on progression of coronary atherosclerosis 
and arterial remodeling: A pooled analysis of 5 intravascular ultrasound trials. J Am Coll Cardiol 2008; 52: 255-262.

24. Garg P, Normand SL, Silbaugh TS, Wolf RE, Zelevinsky K, Lovett A, et al. Drug-eluting or bare-metal stenting in patients with diabetes mellitus: Results from the Massachusetts Data Analysis Center Registry. Circulation 2008; 118: 2277-2285, 7p following 85.

25. Buse JB, Ginsberg HN, Bakris GL, Clark NG, Costa F, Eckel R, et al; American Heart Association; American Diabetes Association. Primary prevention of cardiovascular diseases in people with diabetes mellitus: A scientific statement from the American Heart Association and the American Diabetes Association. Circulation 2007; 115: 114-126.

26. Yamagata K, Kataoka Y, Kokubu N, Kasahara Y, Abe M, Nakajima $\mathrm{H}$, et al. A 3-year clinical outcome after percutaneous coronary intervention using sirolimus-eluting stent and off-pump coronary artery bypass grafting for the treatment of diabetic patients with multivessel disease. Circ J 2010; 74: 671-678.
27. Kim KS. PCI vs CABG in complicated diabetic patients: Do we need more data to change the practical guideline? Circ J 2010; 74: $622-623$.

28. Smith SC Jr, Blair SN, Criqui MH, Fletcher GF, Fuster V, Gersh BJ, et al. AHA consensus panel statement: Preventing heart attack and death in patients with coronary disease: The Secondary Prevention Panel. J Am Coll Cardiol 1995; 26: 292-294.

29. Maron DJ, Boden WE, O'Rourke RA, Hartigan PM, Calfas KJ, Mancini GB, et al; COURAGE Trial Research Group. Intensive multifactorial intervention for stable coronary artery disease: Optimal medical therapy in the COURAGE (Clinical Outcomes Utilizing Revascularization and Aggressive Drug Evaluation) trial. $J \mathrm{Am}$ Coll Cardiol 2010; 55: 1348-1358.

30. Zhang B, Matsunaga A, Rainwater DL, Miura S, Noda K, Nishikawa $\mathrm{H}$, et al. Effects of rosuvastatin on electronegative LDL as characterized by capillary isotachophoresis: The ROSARY Study. J Lipid Res 2009; 50: 1832-1841. 